\title{
Suppression of LPS-induced inflammatory responses in macrophages infected with Leishmania
}

Nicholas J Lapara III, Ben L Kelly

\begin{abstract}
Background: Chronic inflammation activated by macrophage innate pathogen recognition receptors such as TLR4 can lead to a range of inflammatory diseases, including atherosclerosis, Crohn's disease, arthritis and cancer. Unlike many microbes, the kinetoplastid protozoan pathogen Leishmania has been shown to avoid and even actively suppress host inflammatory cytokine responses, such as LPS-induced IL-12 production. The nature and scope of Leishmania-mediated inflammatory cytokine suppression, however, is not well characterized. Advancing our knowledge of such microbe-mediated cytokine suppression may provide new avenues for therapeutic intervention in inflammatory disease.

Methods: We explored the kinetics of a range of cytokine and chemokine responses in primary murine macrophages stimulated with LPS in the presence versus absence of two clinically distinct species of Leishmania using sensitive multiplex cytokine analyses. To confirm that these effects were parasite-specific, we compared the effects of Leishmania uptake on LPS-induced cytokine expression with uptake of inert latex beads.

Results: Whilst Leishmania uptake alone did not induce significant levels of any cytokine analysed in this study, Leishmania uptake in the presence of LPS caused parasite-specific suppression of certain LPS-induced proinflammatory cytokines, including IL-12, IL-17 and IL-6. Interestingly, L. amazonensis was generally more suppressive than L. major. We also found that other LPS-induced proinflammatory cytokines, such as IL-1 $\alpha$, TNF- $\alpha$ and the chemokines MIP- $1 \alpha$ and MCP-1 and also the anti-inflammatory cytokine IL-10, were augmented during Leishmania uptake, in a parasite-specific manner.

Conclusions: During uptake by macrophages, Leishmania evades the activation of a broad range of cytokines and chemokines. Further, in the presence of a strong inflammatory stimulus, Leishmania suppresses certain proinflammatory cytokine responses in a parasite-specific manner, however it augments the production of other proinflammatory cytokines. Our findings highlight the complexity of inflammatory cytokine signalling regulation in the context of the macrophage and Leishmania interaction and confirm the utility of the Leishmania/macrophage infection model as an experimental system for further studies of inflammatory regulation. Such studies may advance the development of therapies against inflammatory disease.
\end{abstract}

\section{Background}

Inflammatory diseases such as atherosclerosis are often caused by chronic inflammation and encompass a large spectrum of diseases, including atherosclerosis, Crohn's disease, arthritis and cancer [1-6]. Persistence of, or repeated exposure to certain microbial pathogens, leads

\footnotetext{
* Correspondence: bkell2@lsuhsc.edu

Department of Microbiology Immunology and Parasitology, LSU Health Sciences Center, 1901 Perdido Street, New Orleans, LA 70112, USA
}

to chronically elevated levels of several etiologic inflammatory mediators, including the cytokines IL-12, tumor necrosis factor alpha (TNF- $\alpha$ ), interferon-gamma (IFN$\gamma)$, IL- 6 and IL-17 $[1,7]$, that are considered to contribute to the onset of inflammatory diseases. Production of these cytokines typically ensues via host cell signalling cascades following the engagement of innate pathogenassociated molecular pattern (PAMP) receptors including the Toll-like receptors (TLRs) expressed primarily by cells of the innate immune compartment, by

\section{() Biomed Central}


pathogen-specific ligands, such as bacterial lipopolysaccharide (LPS) [8]. Importantly, recent studies with geneknockout mice and also human TLR polymorphisms have confirmed that TLR signaling in response to pathogen contact can also contribute to the development of atherosclerosis $[9,10]$. The mechanisms that underlie inflammatory cytokine induction following engagement of the TLR receptors have been well studied for a number of cytokines and commonly result in nuclear translocation of nuclear factor kappa-light-chain-enhancer of activated B cells (NF- $\kappa \mathrm{B})$, a Rel-family transcription factor, followed by its binding to DNA sequences associated with the promoters of their target genes such as IL-12 [11]. In addition to NF- $\kappa \mathrm{B}$ activation, TLR signaling may also activate mitogen activated protein (MAP) kinase pathways that lead to activation of the transcription factor activating protein 1 (AP-1) and interferon regulatory factor (IRF) signalling [1].

In contrast, whilst macrophage IL-17 production has been demonstrated to be important in allergic inflammation [7], pathways that culminate in the regulation of this proinflammatory cytokine in macrophages, however, have not yet been characterized.

Unlike most microbial pathogens, insect stages (promastigotes) of the protozoan trypanosomatid parasite Leishmania enter macrophage host cells in a way that eludes immediate "classical" proinflammatory activation. Furthermore, a number of studies have shown that upon LPS stimulation of macrophages, IL-12 is actively suppressed by Leishmania [12,13]. Leishmania majorinfected C57/BL6 mice, also show an initial "silent" phase of parasite replication that persists in the dermis for up to 5 weeks [14] prior to disease resolution, indicating parasite-mediated immune evasion occurs in vivo. Experimental infections with $L$. amazonensis also show early impairment of inflammatory responses [15]. Importantly, microarray experiments also showed that infection of the human monocyte line, THP-1, with Leishmania suppressed the IFN $\gamma$-induced expression of many host genes [16].

Cameron et al., [12] showed that the suppression of LPS-induced IL-12 by L. mexicana correlated with degradation of the innate immune signaling MAP kinases JNK and ERK, and also components of the NF$\kappa \mathrm{B}$ signaling pathway, indicating that Leishmania may promote a generalized abrogation of the inflammatory response.

Additional mechanisms that have been proposed for Leishmania's ability to suppress inflammatory activation include the engagement of suppression-associated macrophage surface receptors such as complement receptor 3 (CR3) and the elaboration of suppressive cytokines such as IL-10 [17,18]. Indeed, studies of macrophages from CR3-deficient mice have confirmed that CR3 engagement is involved in IL-12 suppression during Leishmania infection even in the presence of IFN- $\gamma$ stimulation $[17,19]$.

Although some progress toward understanding how Leishmania suppresses macrophage inflammatory responses has been made, our knowledge of the extent to which Leishmania modulates macrophage cytokine responses and the underlying molecular mechanisms involved, remains limited.

To further our understanding of host inflammatory responses modulated by Leishmania, we have explored the inflammation-suppressive effects of both $L$. major and $L$. amazonensis in the context of macrophage infection during TLR4 stimulation, upon a broader range of cytokines than previously studied. These parasites represent two related but distinct microbes responsible for clinically distinct forms of leishmaniasis. Specifically, we studied the modulatory effects of $L$. major and $L$. amazonensis upon the proinflammatory cytokines IL-17, IL$1 \alpha$ and TNF- $\alpha$, the primary Th1-inducing proinflammatory cytokine, IL-12, and the Th2-associated cytokines IL-4,-13, -6,-10 and IL-3 [20]. We also assayed for the macrophage inflammatory chemokines macrophage inflammatory protein $1 \alpha$ (MIP-1 $\alpha)$ and monocyte chemotactic protein-1 (MCP-1). We find that, although LPS-induced IL-17 and IL-12 are repressed by both Leishmania species, LPS-induced TNF- $\alpha$ and IL- $1 \alpha$ responses are enhanced. In addition, we determined that both Leishmania species suppress LPS-induced IL-6, -13 and -3 . Furthermore, L. amazonensis also suppressed LPS-induced IL-4 and IL-10 whereas both Leishmania species augmented LPS-induced MIP- $1 \alpha$ and MCP-1 production. Our findings suggest, at least in the context of TLR4 stimulation, that Leishmania promastigotes do not promote generalized proinflammatory suppression and instead appear to target specific cytokine signalling pathways downstream of the TLR4 receptor, to selectively modulate cytokine and chemokine production during macrophage parasitization.

\section{Methods}

\section{Isolation and Culture of Peritoneal Macrophages}

Peritoneal cells were isolated by lavage from C57/BL6 mice that were purchased from The Jackson Laboratory essentially as described previously [21], with the exception that RPMI-1640/10\% FBS was used for peritoneal lavage.

The peritoneal cells were stained with macrophage markers F4/80, CD14, CD11b and CD 205 then analysed by flow cytometry to confirm their macrophage phenotype and adjusted to a cell density of $4 \times 10^{5} / \mathrm{ml}$.

\section{Parasites}

Promastigotes of $L$. major strain WHOM/IR/-/173 and L. amazonensis strain IFLA/BR/67/PH8 (kindly provided 
by Dr David L. Sacks, NIAID, Bethesda, MD) were cultured in vitro at $27^{\circ} \mathrm{C}$ in medium 199 with $10 \%$ heatinactivated FBS as previously described [22]. Stationary phase parasites were centrifuged at $1300 \times \mathrm{g}$ and resuspended in RPMI-1640 culture medium supplemented with $10 \%$ FBS prior to addition to macrophage monolayers.

\section{Macrophage Treatment}

$0.8 \mathrm{ml}$ aliquots of macrophages $\left(4 \times 10^{5} / \mathrm{ml}\right)$ were incubated for $2 \mathrm{hr}$ on 4-well glass chamber slides, washed to remove non-adherent cells, then co-incubated with or without LPS $(100 \mathrm{ng} / \mathrm{ml})$, followed by the addition of stationary phase promastigotes (20:1 parasite: macrophage), or $6 \mu \mathrm{m}$ latex beads (Sigma) (20:1 bead: macrophage), as indicated in the Results. At 2, 8 or $19 \mathrm{hr}$ timepoints, $200 \mu \mathrm{l}$ of culture supernatant was removed and centrifuged to remove particulates prior to multiplex cytokine analysis.

Following removal of the $19 \mathrm{hr}$ culture supernatants, the macrophages were washed twice with PBS and stained using Diff-Quik (DADE-Behring) as described previously [22], evaluated microscopically (approximately 200 fields observed) and infection/uptake rates determined to be $74 \%, 79 \%$ and $82 \%$ for $L$. major and $L$. amazonensis, and latex beads respectively.

\section{Multiplex cytokine analysis}

Multiplex cytokine analyses that were performed using a Bio-Plex kit (Bio-Rad) in accordance with manufacturer's instructions and analysed using a Luminex machine (Luminex Corporation).

\section{ELISA assays}

Duplicate culture supernatants were removed at the 19 hr timepoint and assayed for cytokine production using ELISA kits from R\&D systems, in accordance with manufacturers' instructions.

\section{Results}

Leishmania suppresses IL-17, IL-12 and IL-3 following induction with LPS

Despite recent interest in IL-17, a key cytokine involved in a variety immune responses, including the induction of other cytokines, its production from macrophages in the context of Leishmania infection has not been characterized. We therefore investigated the release of IL-17 from macrophages during infection with Leishmania alone, or during Leishmania infection in the presence of LPS. Although incubation of macrophages with either $L$. major or L. amazonensis alone did not induce IL-17 production, nor significant levels of any other cytokine we assayed, stimulation with LPS alone caused significant IL-17 induction compared to controls, as shown in Figure 1A. However, co-incubation of the macrophages with LPS in the presence of L. major or L. amazonensis resulted in 7.1-fold and 13.1-fold suppression of IL-17, respectively, relative to LPS alone (Figure 1A). To confirm that this suppression was not merely a consequence of non-specific phagocytic uptake, we also analysed IL17 production from LPS in the presence of $6 \mu \mathrm{m}$ latex beads that were of comparable size to Leishmania. In contrast to incubation with LPS and Leishmania, coincubation of LPS with latex beads caused less than two-fold suppression compared to LPS alone (Figure 1A, white bars), indicating these effects were Leishmania-specific. Since L. mexicana has previously been shown to suppress LPS-induced IL-12 production [12], we also analysed the macrophage LPS-induced IL-12 response in the presence of $L$. major and L. amazonensis, or latex beads as a non-specific phagocytosis control. As shown in Figure 1B, L. major and L. amazonensis suppressed peak levels of LPS-induced IL-12 p40 subunit 3.3-fold and 4.7-fold respectively, supporting previous findings [12]. In contrast, latex beads suppressed IL-12p40 production only 1.4 -fold. Since it is the IL12 p70 heterodimer (IL-12p40/IL-12p35) that induces Th1 responses, we also sought to compare its regulation with IL-12p40. Although less striking than the downregulation of IL-12 p40, Leishmania amazonensis mediated 2-fold down-regulation of LPS-induced IL12 p70 at 19 hrs, whereas L. major and latex beads, both showed modest suppression (Figure 1C). We also investigated the effects of Leishmania upon the induction of IL-3 since IL-3 production may promote intracellular survival because it has been associated with differentiation of monocytes into macrophages that are less responsive to IFN $\gamma$ than macrophages differentiated with GM-CSF [23]. As shown in Figure 1D, whilst LPS induced IL-3 levels significantly above the control treatments, L. major and L. amazonensis suppressed LPSinduced IL-3 2.5-fold and 3.4-fold respectively. In contrast, treatment with LPS and latex beads caused 1.4fold suppression, indicating that the IL-3 suppressive effects of Leishmania were specific.

Leishmania suppresses the Th2-associated cytokines IL-4, IL-6 and IL-13 following induction with LPS

Since susceptibility to Leishmania infection is generally associated with a Th2 response in infected mice, we investigated the effect of Leishmania on the ability of LPS to induce the Th2 cytokines IL-4, IL-6, and IL-13 from macrophages, the primary cells parasitized by Leishmania promastigotes. As shown in Figure 2A, whilst LPS-induced production of IL-4 was significantly upregulated by LPS alone (Figure 2A), peak production of LPS-induced IL-4 was suppressed 2.3-fold by L. amazonensis and 1.4-fold by latex beads, whereas negligible suppression of LPS-induced IL-4 was observed for $L$. major (1.2-fold suppression). In the presence of $L$. major, L. amazonensis and latex beads, LPS-induced IL6 responses were substantially abrogated, with 2.7 -fold, 


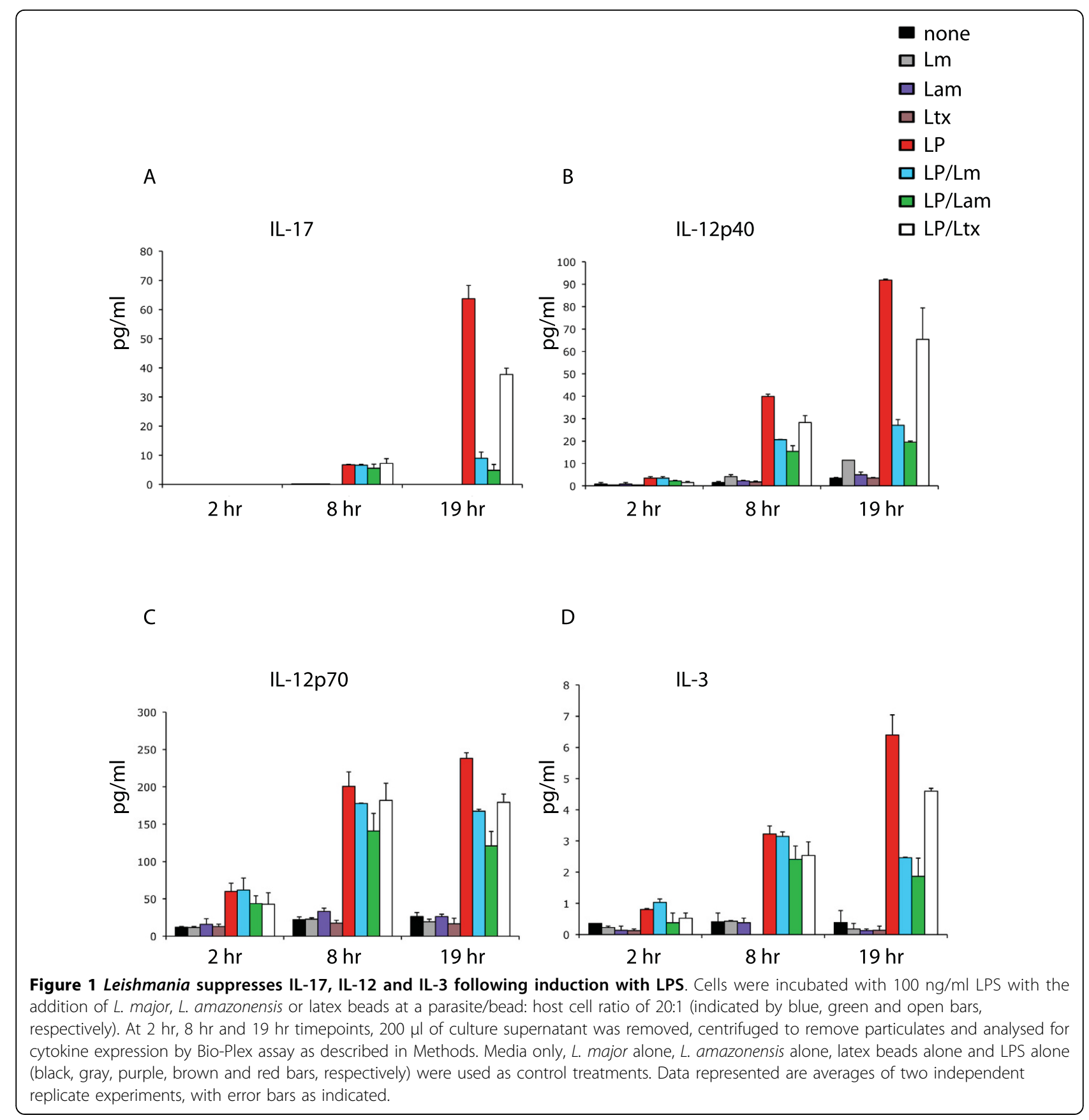

4.8-fold and 1.8-fold reductions respectively. Whilst LPS induced significant levels of IL-13, L. major, L. amazonensis and latex beads showed modest suppression, down regulating LPS-induced IL-13 1.5 fold, 2-fold and 1.3-fold respectively (Figure $2 \mathrm{~B}$ ).

Leishmania augments release of IL-1 $\alpha$, TNF $\alpha$ and IL-10 following LPS induction

To determine if the suppressive effects of Leishmania reflected a generalized counter-inflammatory response against LPS-induced proinflammatory macrophage cytokines, we also analysed the effect of Leishmania uptake upon LPS-induction of the proinflammatory cytokines IL- $1 \alpha$ and TNF $\alpha$. As shown in Figure 3A, we found that, in contrast to its suppressive effects on IL17 and IL-12, L. major augmented LPS-induced IL-1 $\alpha$ 1.6-fold. Conversely L. amazonensis had no effect on LPS-induced IL-1 $\alpha$ levels and latex beads showed a 1.7fold suppressive effect. In contrast to IL-1 $\alpha$, both $L$. major and L. amazonensis augmented LPS-induced TNF $\alpha 1.8$ - and 1.9-fold, respectively at $8 \mathrm{hrs}$, the peak 


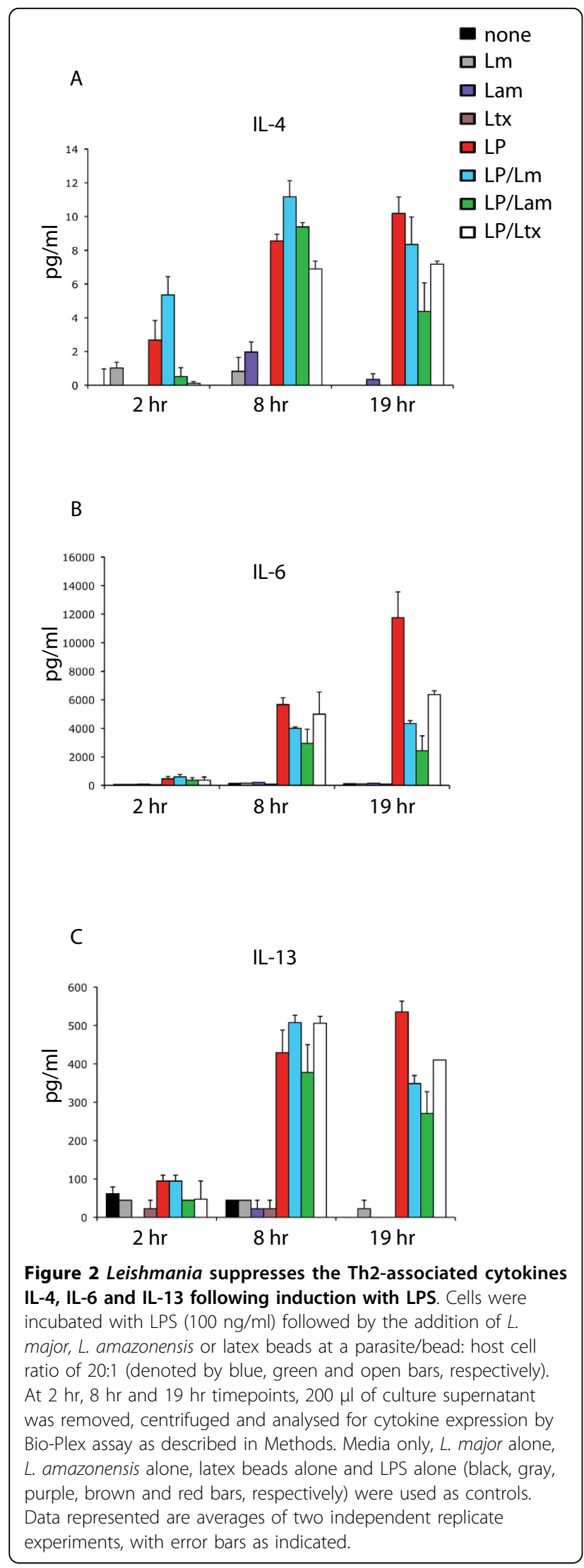

of the LPS induced TNF $\alpha$ response (Figure 3B). These data demonstrate that although Leishmania suppresses certain LPS-induced proinflammatory cytokines, it can simultaneously facilitate the production of other classical inflammatory cytokines. Interestingly, L. amazonensis generally showed a more suppressive effect than $L$. major.

We also investigated the impact of Leishmania uptake on LPS-induced IL-10, since this cytokine typically antagonizes the biological effects of classic proinflammatory cytokines such as IL-12. As shown in Figure 3C, at $8 \mathrm{hr}$ Leishmania augmented LPS-induced IL-10, however by $19 \mathrm{hrs}$, the peak of the LPS-induced IL-10 response, the levels of IL-10 were not significantly different between LPS alone and both Leishmania species. In contrast, latex beads showed moderate suppression of LPS-induced IL-10 at this timepoint.

Leishmania promotes the production of LPS-induced MIP$1 \alpha$ and MCP-1

Since the chemokines macrophage inflammatory protein (MIP) $-1 \alpha$ and macrophage chemoattractant protein (MCP)-1 have been shown to have an important role in limiting macrophage parasitic burden [24] we sought to determine whether Leishmania could suppress expression of these chemokines as a possible way to enhance parasitization. As shown in Figure 4A, at the peak of the LPS-induced response, L. major and L. amazonensis promoted LPS-induced MIP-1 $\alpha$ 1.7-fold and 1.6-fold respectively, whereas latex beads had little effect. Analysis of LPS-induced MCP-1 showed that $L$. major and $L$. amazonensis dramatically augmented LPS-induced levels of this chemokine, with 4.7-fold and 3.6-fold upregulation respectively, whilst treatment of the LPS-induced macrophages with latex beads showed no significant impact on MCP-1 levels (Figure 4B). Both L. major and L. amazonensis were also found to suppress LPSinduced MIP-1 $\beta$ (data not shown).

Our findings were further validated by sampling cytokine levels using ELISA assays, as shown for IL-17 (Figure 5; data not shown).

\section{Discussion}

Macrophages are key mediators of inflammatory responses that are important for host immune protection against infectious challenge. When such responses become dysregulated or chronically activated, however, they contribute to the development of inflammatory diseases such as atherosclerosis and arthritis. Although significant advances, such as the identification of TLRs and their associated intracellular signalling pathways, have contributed substantially to our understanding of how inflammatory responses are activated, critical gaps remain in our understanding of how some microbes avoid and even suppress host inflammatory responses. 


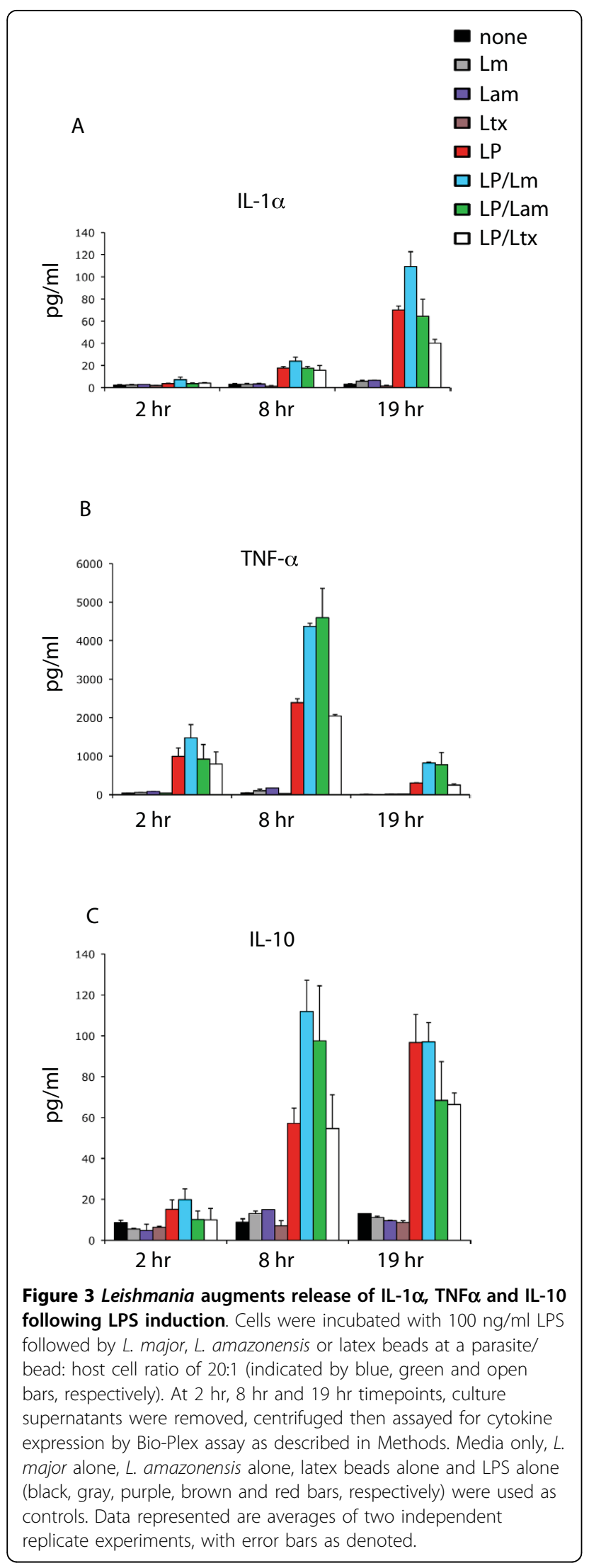

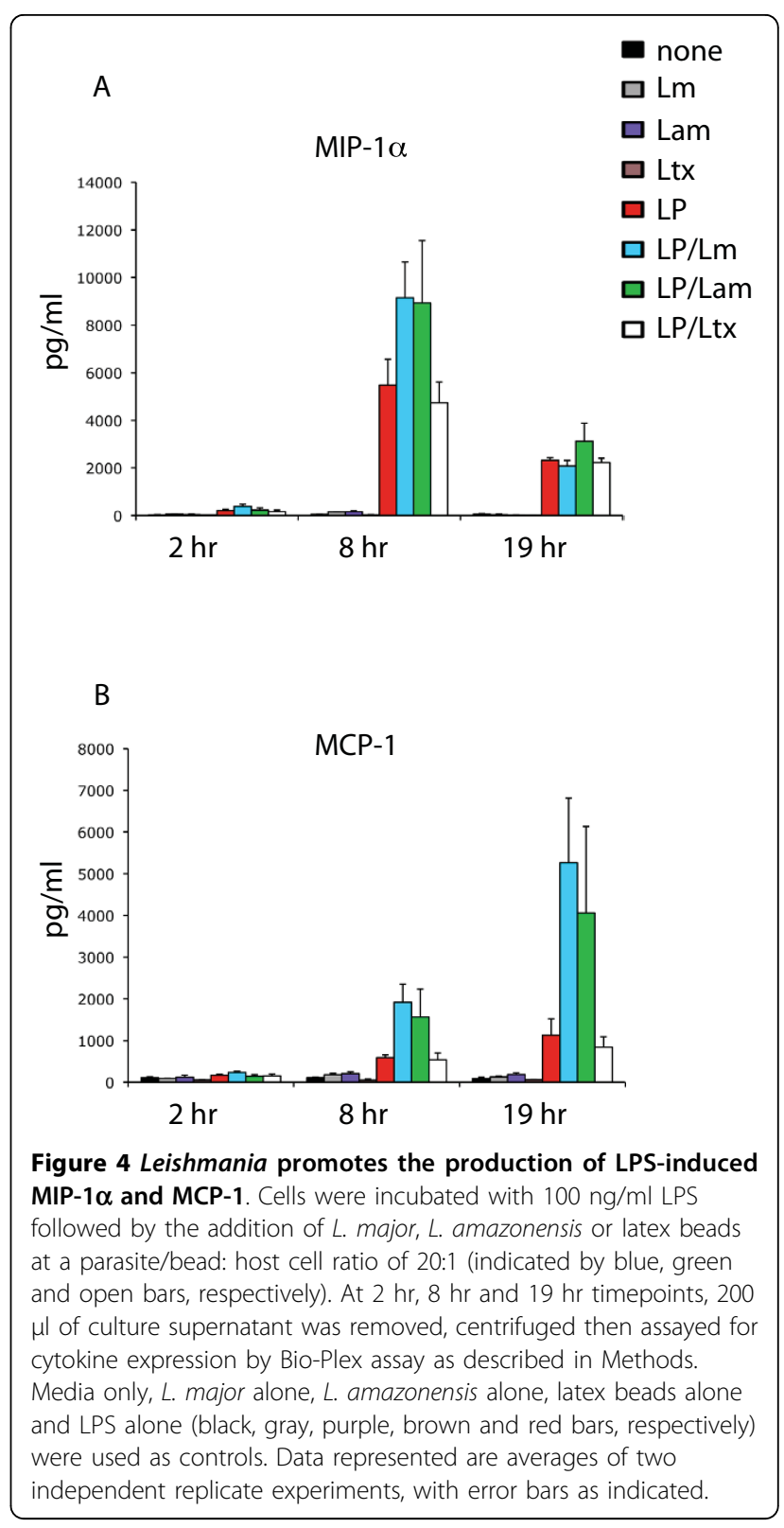

Previous studies have identified Leishmania as a eukaryote protozoan microbe that circumvents the classical host innate immune response, and also actively suppresses macrophage responses to strong inflammatory stimuli such as bacterial LPS [12,25-27]. These studies have typically been limited to analyses of a relatively low number of cytokines, and in general have not distinguished between the specific effects of Leishmania uptake and non-specific effects of generalized phagocytosis upon LPS-induced macrophage activation. We sought to further explore the modulatory effects of Leishmania upon LPS-induced macrophages by determining the specific effects of two clinically distinct species of Leishmania upon the kinetics of a broader 


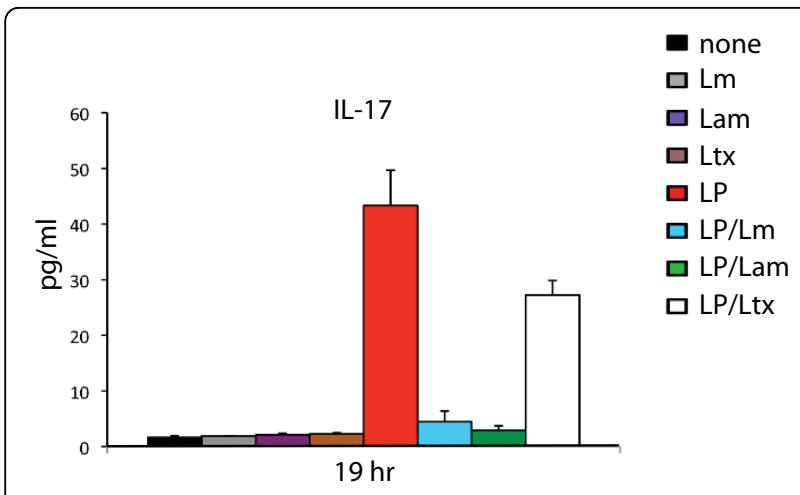

Figure 5 ELISA analysis of Leishmania-mediated cytokine suppression. Cells were incubated with LPS $(100 \mathrm{ng} / \mathrm{ml})$ followed by L. major, L. amazonensis or latex beads at a parasite/bead to host cell ratio of 20:1 (indicated by blue, green and open bars, respectively). At $19 \mathrm{hrs}$, culture supernatants were taken, centrifuged then assayed for IL-17 production by ELISA as described in Methods. Media only, L. major alone, L. amazonensis alone, latex beads alone and LPS alone (black, gray, purple, brown and red bars, respectively) were used as controls. Data represented are averages of two independent replicate experiments, with error bars as denoted.

repertoire of cytokine and chemokine responses elicited by LPS-activated macrophages.

Consistent with previous observations, our studies demonstrate that Leishmania efficiently evades robust activation of many cytokines and chemokines. In the context of LPS-induction, we found that the proinflammatory cytokines IL-17, IL-12 and IL-3, and the Th2 cytokines IL4, IL-6 and IL-13 were all suppressed to varying degrees by both $L$. major and L. amazonesis. For each of these cytokines, the suppressive effects were more pronounced with $L$. amazonensis than L. major. Although we cannot rule out the possibility that the increased suppressive effects we observed for $L$. amazonensis were due to their marginally increased level of uptake compared to L. major, we feel it is unlikely that such a subtle increase in parasite uptake would lead to the striking increases in cytokine suppression we observed.

Although the latex bead controls were moderately suppressive at the peak of the LPS response, we found that in general, Leishmania were significantly more so. Another interesting finding of our study was that whilst Leishmania suppressed some LPS-induced responses, such as IL-4 and IL-13 at timepoints when the LPS response was maximal (usually $19 \mathrm{hrs}$ ), these responses were actually augmented by Leishmania at the $8 \mathrm{hr}$ timepoint. This observation is reminiscent of the refractory period observed with Toxoplasma gondii-induced IL-12 production in dendritic cells [28], and may therefore not represent true suppression.

Although IL-17-producing Th17 cells have been the subject of intensive study recently and IL-17 production is critical for the expansion of innate immune cells, only one report has described the importance of macrophage-derived IL-17 in inflammatory disease [7]. Indeed, our study is the first to investigate the impact of Leishmania upon LPS-induced IL-17 production in macrophages. Our observations that both $L$. major and $L$. amazonensis promastigotes strongly and specifically suppress LPS-induced IL-17 production, provide a new model for studying mechanisms of IL-17 regulation.

Although we observed suppression of both IL-12p40 and IL-12p70, IL-12p40 suppression was more striking. Interestingly, our finding that L. major and latex beads had similar mildly suppressive effects suggests that phagocytic uptake alone contributes significantly to suppression of LPS-induced IL-12p70 during L. major infection. Our findings with $L$. amazonensis differ from those of Cameron et al., [12], which showed its close relative, $L$. mexicana, caused a more dramatic suppression of LPSinduced IL-12p70. Instead, our data are more comparable to previous findings with $L$. major-infected human monocytes [29]. These different observations may be attributable to distinct pathobiological properties known to exist even amongst closely related Leishmania species.

L. major but not L. amazonensis showed augmentation of LPS-induced IL-1 $\alpha$. Interestingly, IL-1 $\alpha$ can promote Th1 responses that ameliorate disease progression in susceptible BALB/c [30] whilst Th1-biased C57BL/6 mice that are resistant to $L$. major remain susceptible to $L$. amazonensis [15]. Whether or not the differences in macrophage responses elicited by $L$. amazonensis versus $L$. major under our conditions contribute to the increased severity of disease typically associated with $L$. amazonensis compared to $L$. major, remains to be determined.

Production of IL-10 by Leishmania alone was not strikingly increased above controls, however at $8 \mathrm{hrs}$ post treatment, both Leishmania species significantly augmented LPS-induced levels of IL-10. Since IL-10 suppresses production of inflammatory cytokines, such as IL-12, it is interesting that a classical proinflammatory stimulus such as LPS also induces IL-10. Indeed, these data are consistent with recent findings [31] and may reflect IL-10-mediated negative feedback regulation. Since IL-10 is a strong suppressor of host cytokine production and promotes disease progression in leishmaniasis $[32,33]$, it is possible that the cytokine-suppressive effects we observed could be attributable to Leishmaniaaugmented IL-10 production. We feel this is unlikely however, since although IL-10 is a potent suppressor of macrophage TNF- $\alpha$ [34], the increased levels of IL-10 released by LPS/Leishmania in our study did not prevent augmentation of TNF $\alpha$ production. Furthermore, in contrast to both $L$. major and L. mexicana, absence of IL-10 does not protect against $L$. amazonensis 
infection [35], suggesting that L. amazonensis employs IL-10-independent immunomodulatory mechanisms.

Although these studies provide new insight into the regulation of macrophage inflammatory responses by Leishmania, it should be emphasized that they were performed in vitro using peritoneal macrophages to identify novel mechanisms of macrophage inflammatory modulation. How these in vitro findings translate to in vivo models of Leishmania infection has not yet been clarified. It is possible, for example, that peritoneal macrophages may behave differently to the inflammatory macrophages found at infection sites in the skin during Leishmania inoculation. Further, the metacyclicenriched promastigote population inoculated during natural infection may have different effects upon macrophage responses than the less-defined heterogenous population of stationary phase parasites used in our studies. It is also important to emphasize that Leishmania amastigote stages may elicit different macrophage responses than promastigotes. As such, future studies with purified metacylic organisms and distinct macrophage populations will be helpful in determining the extent to which our novel findings pertain to Leishmania infection in vivo.

We also emphasize that studies of promastigotemediated modulation of LPS-induced cytokine responses may have limited relevance in the context of natural Leishmania infections, since Leishmania possesses no known TLR4 agonists and there are no data confirming that TLR4 agonists are introduced into the sandfly bite site. Instead, however, these studies may be relevant for the design of protective adjuvant-based vaccination strategies against Leishmania, using TLR agonists to promote optimal inflammatory cytokine profiles that may facilitate sustained Th1 responses required for protective immunity against this pathogen.

\section{Conclusions}

We have explored the cytokine-modulatory effects of two clinically distinct species of Leishmania upon the kinetics of a range of LPS-induced macrophage inflammatory responses. We find both suppression and promotion of LPS-induced responses, indicating the selective suppression and augmentation of specific cytokine induction mechanisms. Our studies provide foundations to pursue functional studies to further elucidate the molecular mechanisms that underlie the distinct cytokine responses to Leishmania that we observed. Such investigations of Leishmania-mediated modulation of host cytokine responses will advance our understanding of inflammatory responses and likely promote new avenues for therapeutic intervention against inflammatory diseases.

\section{Abbreviations}

TLR: Toll-like receptor; NF- $\kappa$ B: nuclear factor kappa-light-chain enhancer of activated B cells; CR3: complement receptor 3; IL-: interleukin; MIP-: macrophage inflammatory protein; MCP-1: macrophage chemotactic protein-1.

\section{Acknowledgements}

We thank Dr. Ashok Aiyar for scientific discussions and Dr. Ronald B. Luftig for critiquing the manuscript. This work was supported by a National Institutes of Health COBRE grant (NIH NCRR P20 RR018766).

\section{Authors' contributions}

NJL executed all experiments and assisted BLK in the experimental design. BLK was responsible for the experimental design and writing the

manuscript. Both Authors have read and approved the final manuscript.

\section{Competing interests}

The authors declare that they have no competing interests.

Received: 13 November 2009

Accepted: 2 February 2010 Published: 2 February 2010

\section{References}

1. Lundberg AM, Hansson GK: Innate immune signals in atherosclerosis. Clin immunol 2010, 134(1):5-24.

2. Arnett HA, Viney JL: Gatekeepers of intestinal inflammation. Inflamm Res 2010, 59(1):1-14.

3. Brand S: Crohn's disease: Th1, Th17 or both? The change of a paradigm: new immunological and genetic insights implicate Th17 cells in the pathogenesis of Crohn's disease. Gut 2009, 58(8):1152-1167.

4. Gersemann M, Wehkamp J, Fellermann K, Stange EF: Crohn's diseasedefect in innate defence. World J Gastroenterol 2008, 14(36):5499-5503.

5. Finckh A: Early inflammatory arthritis versus rheumatoid arthritis. Current opinion in rheumatology 2009, 21(2):118-123.

6. Gonda TA, Tu S, Wang TC: Chronic inflammation, the tumor microenvironment and carcinogenesis. Cell cycle 2009, 8(13):2005-2013.

7. Song C, Luo L, Lei Z, Li B, Liang Z, Liu G, Li D, Zhang G, Huang B, Feng ZH: IL-17-producing alveolar macrophages mediate allergic lung inflammation related to asthma. J Immunol 2008, 181(9):6117-6124.

8. Trinchieri $\mathrm{G}$, Sher $\mathrm{A}$ : Cooperation of Toll-like receptor signals in innate immune defence. Nature reviews 2007, 7(3):179-190.

9. Michelsen KS, Doherty TM, Shah PK, Arditi M: TLR signaling: an emerging bridge from innate immunity to atherogenesis. J Immunol 2004, 173(10):5901-5907.

10. Cook DN, Pisetsky DS, Schwartz DA: Toll-like receptors in the pathogenesis of human disease. Nature immunology 2004, 5(10):975-979.

11. Murphy TL, Cleveland MG, Kulesza P, Magram J, Murphy KM: Regulation of interleukin 12 p40 expression through an NF-kappa B half-site. Molecular and cellular biology 1995, 15(10):5258-5267.

12. Cameron P, McGachy A, Anderson M, Paul A, Coombs GH, Mottram JC, Alexander J, Plevin R: Inhibition of lipopolysaccharide-induced macrophage IL-12 production by Leishmania mexicana amastigotes: the role of cysteine peptidases and the NF-kappaB signaling pathway. J Immunol 2004, 173(5):3297-3304.

13. Belkaid $Y$, Butcher B, Sacks DL: Analysis of cytokine production by inflammatory mouse macrophages at the single-cell level: selective impairment of IL-12 induction in Leishmania-infected cells. European journal of immunology 1998, 28(4):1389-1400.

14. Belkaid Y, Mendez S, Lira R, Kadambi N, Milon G, Sacks D: A natural model of Leishmania major infection reveals a prolonged "silent" phase of parasite amplification in the skin before the onset of lesion formation and immunity. J Immunol 2000, 165(2):969-977.

15. Ji J, Sun J, Soong L: Impaired expression of inflammatory cytokines and chemokines at early stages of infection with Leishmania amazonensis. Infect Immun 2003, 71(8):4278-4288.

16. Dogra N, Warburton C, McMaster WR: Leishmania major abrogates IFN\{gamma\} induced gene expression in human macrophages on a global scale. Infect Immun 2007, 75(7):3506-15.

17. Schonlau F, Scharffetter-Kochanek K, Grabbe S, Pietz B, Sorg C, Sunderkotter $C$ : In experimental leishmaniasis deficiency of $C D 18$ results in parasite dissemination associated with altered macrophage functions 
and incomplete Th1 cell response. European journal of immunology 2000, 30(9):2729-2740.

18. Von Stebut E: Immunology of cutaneous leishmaniasis: the role of mast cells, phagocytes and dendritic cells for protective immunity. Eur J Dermatol 2007, 17(2):115-122.

19. Carter CR, Whitcomb JP, Campbell JA, Mukbel RM, McDowell MA: Complement Receptor 3-deficiency influences lesion progression during Leishmania major infection in BALB/c mice. Infect Immun 2009, 77(12):5668-5675.

20. Aoki I, Tanaka S, Ishii N, Minami M, Klinman DM: Contribution of interleukin-3 to antigen-induced Th2 cytokine production. European journal of immunology 1996, 26(6):1388-1393.

21. Stapleton PP, Fujita J, Murphy EM, Naama HA, Daly JM: The influence of restricted calorie intake on peritoneal macrophage function. Nutrition 2001, 17(1):41-45.

22. Kelly BL, Stetson DB, Locksley RM: Leishmania major LACK antigen is required for efficient vertebrate parasitization. The Journal of experimental medicine 2003, 198(11):1689-1698.

23. Saha B, Saini A, Germond R, Perrin PJ, Harlan DM, Davis TA: Susceptibility or resistance to Leishmania infection is dictated by the macrophages evolved under the influence of IL-3 or GM-CSF. European journal of immunology 1999, 29(7):2319-2329.

24. Dey R, Majumder N, Bhattacharyya Majumdar S, Bhattacharjee S, Banerjee S, Roy $S$, Majumdar $S$ : Induction of host protective Th1 immune response by chemokines in Leishmania donovani-infected BALB/C mice. Scandinavian journal of immunology 2007, 66(6):671-683.

25. Weinheber N, Wolfram M, Harbecke D, Aebischer T: Phagocytosis of Leishmania mexicana amastigotes by macrophages leads to a sustained suppression of IL-12 production. European journal of immunology 1998, 28(8):2467-2477.

26. Ben-Othman R, Guizani-Tabbane L, Dellagi K: Leishmania initially activates but subsequently down-regulates intracellular mitogen-activated protein kinases and nuclear factor-kappaB signaling in macrophages. Molecular immunology 2008, 45(11):3222-3229.

27. Ben-Othman R, Dellagi K, Guizani-Tabbane L: Leishmania major parasites induced macrophage tolerance: implication of MAPK and NF-kappaB pathways. Molecular immunology 2009, 46(16):3438-3444.

28. Reis e Sousa C, Yap G, Schulz O, Rogers N, Schito M, Aliberti J, Hieny S, Sher A: Paralysis of dendritic cell IL-12 production by microbial products prevents infection-induced immunopathology. Immunity 1999, 11(5):637-647.

29. Meddeb-Garnaoui A, Zrelli H, Dellagi K: Effects of tropism and virulence of Leishmania parasites on cytokine production by infected human monocytes. Clinical and experimental immunology 2009, 155(2):199-206.

30. Von Stebut E, Ehrchen JM, Belkaid Y, Kostka SL, Molle K, Knop J, Sunderkotter C, Udey MC: Interleukin 1alpha promotes Th1 differentiation and inhibits disease progression in Leishmania major-susceptible BALB/C mice. The Journal of experimental medicine 2003, 198(2):191-199.

31. Kang JW, Choi SC, Cho MC, Kim HJ, Kim JH, Lim JS, Kim SH, Han JY, Yoon DY: A proinflammatory cytokine interleukin-32beta promotes the production of an anti-inflammatory cytokine interleukin-10. Immunology 2009, 128(1 Suppl):e532-540.

32. Buxbaum LU, Scott P: Interleukin 10- and Fcgamma receptor-deficient mice resolve Leishmania mexicana lesions. Infect Immun 2005, 73(4):2101-2108.

33. Kane MM, Mosser DM: The role of IL-10 in promoting disease progression in leishmaniasis. J Immunol 2001, 166(2):1141-1147.

34. Denys A, Udalova IA, Smith C, Williams LM, Ciesielski CJ, Campbell J, Andrews C, Kwaitkowski D, Foxwell BM: Evidence for a dual mechanism for IL-10 suppression of TNF-alpha production that does not involve inhibition of p38 mitogen-activated protein kinase or NF-kappa B in primary human macrophages. J Immunol 2002, 168(10):4837-4845.

35. Jones DE, Ackermann MR, Wille U, Hunter CA, Scott P: Early enhanced Th1 response after Leishmania amazonensis infection of C57BL/6 interleukin10-deficient mice does not lead to resolution of infection. Infect Immun 2002, 70(4):2151-2158.

doi:10.1186/1476-9255-7-8

Cite this article as: Lapara and Kelly: Suppression of LPS-induced inflammatory responses in macrophages infected with Leishmania. Journal of Inflammation 2010 7:8.

\section{Submit your next manuscript to BioMed Central and take full advantage of:}

- Convenient online submission

- Thorough peer review

- No space constraints or color figure charges

- Immediate publication on acceptance

- Inclusion in PubMed, CAS, Scopus and Google Scholar

- Research which is freely available for redistribution 